\title{
Spiderweb Central Configurations
}

\author{
Olivier Hénot ${ }^{1}$. Christiane Rousseau ${ }^{1}$ (])
}

Received: 11 October 2018 / Accepted: 19 June 2019 / Published online: 28 June 2019

(c) The Author(s) 2019

\begin{abstract}
In this paper we study spiderweb central configurations for the $N$-body problem, i.e configurations given by $N=n \times \ell+1$ masses located at the intersection points of $\ell$ concurrent equidistributed half-lines with $n$ circles and a central mass $m_{0}$, under the hypothesis that the $\ell$ masses on the $i$-th circle are equal to a positive constant $m_{i}$; we allow the particular case $m_{0}=0$. We focus on constructive proofs of the existence of spiderweb central configurations, which allow numerical implementations. Additionally, we prove by a rigorous numerical method the uniqueness of such central configurations when $\ell \in\{2, \ldots, 9\}$ and arbitrary $n$ and $m_{i}$; under the constraint $m_{1} \geq m_{2} \geq \cdots \geq m_{n}$ we also prove uniqueness for $\ell \in\{10, \ldots, 18\}$ and $n$ not too large. We also give an algorithm providing a rigorous proof of the existence and local unicity of such central configurations when given as input a choice of $n, \ell$ and $m_{0}, \ldots, m_{n}$. Finally, our numerical simulations highlight some interesting properties of the mass distribution.
\end{abstract}

\section{Introduction}

The $N$-body problem consists in describing the positions $\mathbf{r}_{1}(t), \ldots, \mathbf{r}_{N}(t)$ of $N$ masses $m_{1}, \ldots, m_{N}$ interacting through Newton's gravitational law, which are solutions of the system of coupled non-linear equations

$$
m_{i} \ddot{\mathbf{r}}_{i}=-\sum_{j \neq i} \frac{G m_{i} m_{j}\left(\mathbf{r}_{i}-\mathbf{r}_{j}\right)}{\left|\mathbf{r}_{i}-\mathbf{r}_{j}\right|^{3}}=-\frac{\partial U(\mathbf{r})}{\partial \mathbf{r}_{i}}=\mathbf{F}_{i}(\mathbf{r}), \quad U=-\sum_{i<j} \frac{G m_{i} m_{j}}{\left|\mathbf{r}_{i}-\mathbf{r}_{j}\right|}
$$

This research was supported by NSERC in Canada.

$凶$ Christiane Rousseau

rousseac@dms.umontreal.ca

1 Université de Montréal, Montreal, Canada

Birkhäuser 
for $i=1, \ldots, N$, with $\mathbf{r} \in\left\{\left(\mathbf{r}_{1}, \ldots, \mathbf{r}_{N}\right) \in \mathbb{R}^{3 N}: \mathbf{r}_{i} \neq \mathbf{r}_{j}, i \neq j\right\}$, where $G$ denotes the gravitational constant.

Specific solutions, called central configurations, arise when the acceleration of each mass-particle is proportional to the position with the same constant of proportionality (depending on time) for all masses. In this paper we are interested in spiderweb configurations of $N=n \times \ell+1$ masses, where the masses are located at the intersection points of $\ell$ concurrent equidistributed half-lines with $n$ circles of radii $r_{1}<\cdots<r_{n}$, and a central mass $m_{0}$, under the hypothesis that the $\ell$ masses on the $i$-th circle are equal to a positive constant $m_{i}$, while the mass $m_{0}$ is allowed to vanish (see Fig. 1).

The existence of such central configurations have been studied in the literature, often in the special case $m_{0}=0$, starting with Moulton in 1910 ([9]), which settled the case $\ell=2$ as a particular case of $N$ aligned masses. The case $n=1$ has been treated by Maxwell in the 19th century [6]. Later Moeckel and Simo [7] proved the existence and uniqueness of such a configuration in the case $n=2$ and $m_{0}=0$ and the inverse problem was considered in [12,13]. In [5] Llibre and Mello considered the cases of three or four nested polygons for no more than $N=9$ bodies. Corbera, Delgado and Llibre [2] considered the case of $n$ and $\ell$ arbitrary with restrictions on the masses of the type $m_{1} \gg \cdots \gg m_{n}$ for $m_{0}=0$, while Saari treated the general case, releasing the restrictions on the masses with a different method in $[10,11]$. We became interested in the problem and decided to study numerically the distribution



(a) $n=3, \ell=5$

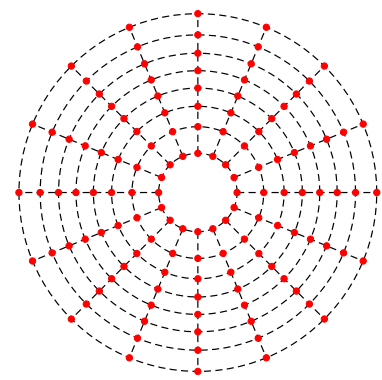

(c) $n=8, \ell=16$

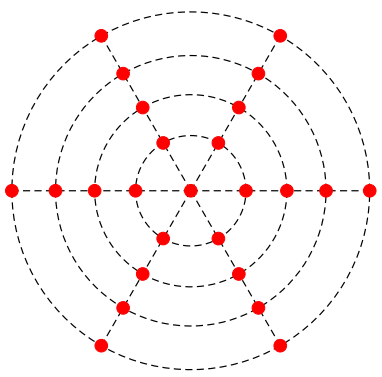

(b) $n=4, \ell=6$

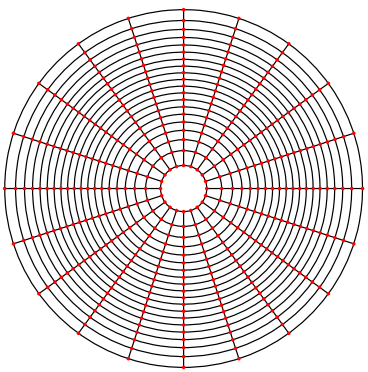

(d) $n=20, \ell=20$

Fig. 1 Spider central configurations with unitary masses. Note that $m_{0} \neq 0$ only in (b) 
$M(\eta)$ of mass depending of the distance $\eta$ to the origin for large values of $\ell$ and $n$. At the same time we considered the proofs of the results appearing in $[2,10,11]$ : to our surprise, these proofs are incomplete and we started working on completing them. We could give some complete proofs, but not for all values of $n, \ell$ and of the masses. But our proofs are constructive and can be implemented numerically. Our numerical experimentations suggest the uniqueness of the central configurations (as claimed by Saari), and allow exploring the mass distribution in these configurations for large values of $n$ and $\ell$. By the time a first version of this paper was ready, we learnt of the general result of Montaldi [8] giving, for any symmetric choice of masses, the existence of central configurations in $\mathbb{R}^{d}$ with any possible symmetry type given by a finite subgroup of $O(d)$ (and hence, in particular, the existence of spiderweb central configurations). The proof of Montaldi, based on a variational formulation of the problem and using the principle of symmetric criticality of Palais, is very elegant. The central configurations are the critical points of the potential energy $U$ on level sets of the total moment of inertia $I$ about the origin. The principle of symmetric criticality of Palais asserts that a critical point of the subset of symmetric configurations is a critical point of $U$. Because $I$ is a positive quadratic form, a minimum of $U$ has to exist on the subset of symmetric configurations, yielding a critical point of $U$. Montaldi's proof is completely existential and gives no hint for the uniqueness of central configurations. Hence we believe that our proofs are a complement to the one provided by Montaldi in [8]. Additionally, for $\ell \in\{2, \ldots, 9\}$ and a few other particular cases, we could prove the uniqueness of the central configurations.

The proof of Corbera, Delgado and Llibre [2] is by induction on $n$. To go from $n$ circles to $n+1$ circles, the idea is to add an $n+1$-th circle with masses $m_{n+1}=0$ and to allow the mass $m_{n+1}$ to increase, via the implicit function theorem. Our proof uses the same idea but is much shorter and straightforward.

By adapting the method of [2] in the spirit of Moulton [9], i.e. starting from a restricted $N$-body problem and following the solutions by the implicit function theorem for large values of the $m_{i}$, we were able to prove in Theorem 4.2 the existence and uniqueness of spiderweb central configurations for $\ell \in\{2, \ldots, 9\}$ and arbitrary $n$ and $m_{i}$. Under constraints on the mass distribution and the maximum number of circles, the uniqueness is also proven in Theorem 4.3 for $\ell \in\{10, \ldots, 18\}$. A technical part of both results is to establish a lower bound for a smooth function on $[0,1]$. Tedious analytical work to obtain this bound has been circumvent by implementing, with interval arithmetics, a numerical method based on the mean value theorem.

In [11], Saari proposed a proof of the existence of spiderweb central configurations in the general case. There, again, the proof was by induction on $n$, and used continuity arguments, which had to rely on the implicit function theorem. But no checking of the hypothesis of the implicit function theorem could be found. Our checking of these hypotheses revealed much harder than expected, but we could adapt the method of Saari and prove in Theorem 5.1 the existence of spiderweb central configurations for arbitrary $\ell$ and $m_{i}$ and $n \in\{3,4\}$.

To conclude, we give an algorithm providing a rigorous proof of the existence and local unicity of such central configurations when given as input a choice of $n, \ell$ and $m_{0}, \ldots, m_{n}$ [3]. The algorithm has been applied to all $n \leq 100$ and $\ell \leq 200$ when $m_{0}=0$ and all masses are equal. We have also applied it in the case of different 
masses. Our numerical explorations allowed studying the profile of the function $M(\eta)$ describing the distribution of mass at the distance $\eta$ from the centre of mass. This profile reveals universal features.

The paper is organized as follows. Section 2 contains preliminaries. Section 3 shows the existence of spiderweb central configurations with $N=n \times \ell+1$ or $N=n \times \ell$, and arbitrary $n$ and $\ell$. In Sect. 4 we prove the existence and uniqueness of spiderweb central configurations for $\ell \in\{2, \ldots, 9\}$, and arbitrary $n$ and $m_{i}$ in the spirit of [9], while in Sect. 5 we give a constructive proof of the existence of spiderweb configurations for $n \in\{3,4\}$ and arbitrary $\ell$. Finally Sect. 6 deals with the numerical algorithm providing rigorous proof of existence, while Sect. 7 studies the properties of the function $M(\eta)$.

\section{Preliminaries}

\subsection{Scalings and Central Configurations in (1.1)}

For simplicity, we translate the center of mass at the origin. Considering changes $(r, m, t) \mapsto(A r, B m, C t)$ in the units of length, mass and time satisfying $A^{3} B^{-1} C^{-2}=G$ scales $G=1$. There remain two degrees of freedom: indeed additional changes preserve $G=1$ provided $A^{3}=B C^{2}$.

Definition 2.1 The configuration of $N$ bodies is central at some time $t^{*}$ if $\ddot{\mathbf{r}}\left(t^{*}\right)=$ $\lambda \mathbf{r}\left(t^{*}\right)$ for some common $\lambda$, where $\mathbf{r} \in\left\{\left(\mathbf{r}_{1}, \ldots, \mathbf{r}_{N}\right) \in \mathbb{R}^{3 N}: \mathbf{r}_{i} \neq \mathbf{r}_{j}, i \neq j\right\}$.

Remark 2.2 The previous definition suggests that being a central configuration is a characteristic of the precise time $t^{*}$. However, it is well-known that, for well-chosen initial velocities, the $N$ bodies remain in a central configuration for all time $t$; during the motion of the $N$ bodies, the common $\lambda$ is a function of $t$.

It is easy to see that $\lambda$ is a strictly negative value given by $\lambda=U / I<0$ where $I=\sum m_{i}\left|\mathbf{r}_{i}\right|^{2}$ is the moment of inertia. A scaling in time allows to take $\lambda=-1$. Keeping $\lambda=-1$, if $m \in \mathbb{R}_{\geq 0}^{N}$ is the vector of masses, then if $(\mathbf{r}, m)$ is a central configuration, so is $\left(A \mathbf{r}, A^{3} m\right)$.

Moreover, by the definition of a central configuration and the equation of motion (1.1), any homothety and rotation of the positions, i.e. $\mathbf{r}=\left(\mathbf{r}_{1}, \ldots, \mathbf{r}_{N}\right) \mapsto A \Omega \mathbf{r}$ where $A>0$ and $\Omega \in S O(3)$, yields a central configuration with $\lambda / A^{3}$. Hence, when discussing the uniqueness of central configurations we mean the uniqueness of the equivalence class for the equivalence relation $(\mathbf{r}, m, \lambda) \equiv\left(A \Omega \mathbf{r}, B m, \frac{\lambda}{A^{3} C^{2}}\right)$ under the condition $A^{3}=B C^{2}$ which we can also write

$$
(\mathbf{r}, m, \lambda) \equiv\left(A \Omega \mathbf{r}, \frac{A^{3} m}{C^{2}}, \frac{\lambda}{A^{3} C^{2}}\right) .
$$

\subsection{Spiderweb Configurations}

We consider spiderweb central configurations formed by $n \times \ell$ masses located at the intersection points of $n$ circles centred at the origin of radii $r_{1}, \ldots, r_{n}$, with $\ell$ half- 
lines starting at the origin, whose angle with the positive $x$-axis is $\theta_{k}=2 \pi k / \ell$ for $k=0, \ldots, \ell-1$, together with a mass $m_{0}$ placed at the origin, under the hypothesis that the $\ell$ masses on the $i$-th circle are equal to a positive constant $m_{i}$.

By symmetry, the gravitational tug is identical for all the masses placed on the same circle. In particular, it is clear that for the mass $m_{0}$ located at the origin $\mathbf{F}_{0} \equiv 0$, so $\ddot{\mathbf{r}}_{0}=\lambda \mathbf{r}_{0}$ is trivially satisfied for any $\lambda$.

Under these considerations, it is sufficient to consider the accelerations of the $n$ bodies on the positive horizontal axis, and the numbers $r_{1}, \ldots, r_{n}$ also denote the positions of the masses on this semi-axis.

Then, the system (1.1) in $\mathbb{R}^{n \ell+1}$ reduces to the following system in $\mathbb{R}^{n}$ :

$$
\ddot{r}_{i}=-\sum_{k=1}^{\ell-1} \frac{m_{i}}{2^{3 / 2} r_{i}^{2}\left(1-\cos \theta_{k}\right)^{1 / 2}}-\frac{m_{0}}{r_{i}^{2}}-\sum_{\substack{j=1 \\ j \neq i}}^{n} \sum_{k=0}^{\ell-1} \frac{m_{j}\left(r_{i}-r_{j} \cos \theta_{k}\right)}{\left(r_{i}^{2}+r_{j}^{2}-2 r_{i} r_{j} \cos \theta_{k}\right)^{3 / 2}}
$$

for $i=1, \ldots, n$, with $\theta_{k}=\frac{2 \pi k}{\ell}$ and $r=\left(r_{1}, \ldots, r_{n}\right) \in \mathcal{R}^{n}$, where

$$
\mathcal{R}^{n}=\left\{r \in \mathbb{R}^{n}: 0<r_{1}<\cdots<r_{n}\right\} .
$$

\subsection{Tools}

Under the previous reduction to (2.2), the contribution of the gravitational force on the mass located at $\left(r_{i}, 0\right)$ is $F_{i}(r)=\sum_{j=1}^{n} F_{i j}\left(r_{i}, r_{j}\right)$, where $F_{i j}\left(r_{i}, r_{j}\right)$ is the contribution of $F_{i}(r)$ coming from the attraction of the $j$-th circle, given by

$$
\frac{F_{i j}\left(r_{i}, r_{j}\right)}{m_{i}}= \begin{cases}-\frac{m_{0}}{r_{i}^{2}}, & j=0, \\ -\sum_{k=1}^{\ell-1} \frac{m_{i}}{2^{3 / 2} r_{i}^{2}\left(1-\cos \theta_{k}\right)^{1 / 2}}, & j=i, \\ -\sum_{k=0}^{\ell-1} \frac{m_{j}\left(r_{i}-r_{j} \cos \theta_{k}\right)}{\left(r_{i}^{2}+r_{j}^{2}-2 r_{i} r_{j} \cos \theta_{k}\right)^{3 / 2}}, & j \neq i \text { and } j>0 .\end{cases}
$$

We introduce

$$
\zeta_{\ell}=\sum_{k=1}^{\ell-1} \frac{1}{\left(1-\cos \theta_{k}\right)^{1 / 2}}
$$

and

$$
\begin{cases}x_{j}=r_{i} / r_{j}, & j>i, \\ y_{j}=r_{j} / r_{i}, & j<i,\end{cases}
$$


where the dependency of $x_{j}, y_{j}$ on $i$ is hidden not to overload the notation.

Then, $F_{i j}\left(r_{i}, r_{j}\right) / m_{i}$ becomes

$$
\frac{F_{i j}}{m_{i}}= \begin{cases}-\frac{m_{0}}{r_{i}^{2}}, & j=0, \\ -\frac{m_{i}}{2^{3 / 2} r_{i}^{2}} \zeta_{\ell}, & j=i, \\ -\frac{m_{j}}{r_{i}^{2}} \sum_{k=0}^{\ell-1} \frac{1-y_{j} \cos \theta_{k}}{\left(1+y_{j}^{2}-2 y_{j} \cos \theta_{k}\right)^{3 / 2}}, & 0<j<i, \\ -\frac{m_{j} x_{j}^{2}}{r_{i}^{2}} \sum_{k=0}^{\ell-1} \frac{x_{j}-\cos \theta_{k}}{\left(1+x_{j}^{2}-2 x_{j} \cos \theta_{k}\right)^{3 / 2}}, & j>i .\end{cases}
$$

Letting

$$
\phi_{v}(x)=\sum_{k=0}^{\ell-1} \frac{1}{d_{k}^{v}(x)}, \quad \text { where } d_{k}(x)=\left(1+x^{2}-2 x \cos \theta_{k}\right)^{1 / 2}
$$

gives

$$
\begin{aligned}
\sum_{k=0}^{\ell-1} \frac{x_{j}-\cos \theta_{k}}{\left(1+x_{j}^{2}-2 x_{j} \cos \theta_{k}\right)^{3 / 2}} & =-\phi_{1}^{\prime}\left(x_{j}\right), \\
\sum_{k=0}^{\ell-1} \frac{1-y_{j} \cos \theta_{k}}{\left(1+y_{j}^{2}-2 y_{j} \cos \theta_{k}\right)^{3 / 2}} & =\sum_{k=0}^{\ell-1} \frac{\left(1+y_{j}^{2}-2 y_{j} \cos \theta_{k}\right)-y_{j}^{2}+y_{j} \cos \theta_{k}}{\left(1+y_{j}^{2}-2 y_{j} \cos \theta_{k}\right)^{3 / 2}} \\
& =\phi_{1}\left(y_{j}\right)+y_{j} \phi_{1}^{\prime}\left(y_{j}\right) .
\end{aligned}
$$

Whence,

$$
\frac{F_{i j}}{m_{i}}= \begin{cases}-\frac{m_{0}}{r_{i}^{2}}, & j=0, \\ -\frac{m_{i}}{2^{3 / 2} r_{i}^{2}} \zeta_{\ell}, & j=i, \\ -\frac{m_{j}}{r_{i}^{2}}\left(\phi_{1}\left(y_{j}\right)+y_{j} \phi_{1}^{\prime}\left(y_{j}\right)\right), & 0<j<i, \\ \frac{m_{j} x_{j}^{2}}{r_{i}^{2}} \phi_{1}^{\prime}\left(x_{j}\right), & j>i,\end{cases}
$$

Lemma 2.3 [7] Let $\phi_{\nu}(x)=\sum_{k=0}^{\ell-1} 1 / d_{k}^{v}(x)$ with $d_{k}(x)=\left(1+x^{2}-2 x \cos \theta_{k}\right)^{1 / 2}$ and $v>0$. Then, for $-1<x<1, \phi_{v}(x)$ is analytic and all the coefficients of its power series expansion are positive. In particular, for $0<x<1, \phi_{1}(x)$ is analytic and all its derivatives are positive. 
Lemma 2.4 Let $F_{i j}$ defined by (2.5) and

$$
\lambda_{i j}=\frac{F_{i j}}{m_{i} r_{i}}
$$

Then

$$
\lambda_{i}=\frac{F_{i}}{m_{i} r_{i}}=\sum_{j=1}^{n} \lambda_{i j}, \quad \text { for } i=1, \ldots, n
$$

We have the following properties:

1.

$$
F_{i j}\left\{\begin{array} { l l } 
{ > 0 , } & { i < j , } \\
{ < 0 , } & { i \geq j , }
\end{array} \quad \text { and } \quad \lambda _ { i j } \left\{\begin{array}{ll}
>0, & i<j \\
<0, & i \geq j
\end{array}\right.\right.
$$

2. $\partial_{r_{j}} \lambda_{i}<0$, for all $i \neq j$.

3. $\partial_{r_{i}} \lambda_{i}>0$, for all $i$.

4. $0>\partial_{r_{k}} \lambda_{i k}>\partial_{r_{k}} \lambda_{j k}$, for all $i<j<k$.

5. $\partial_{r_{k}} \lambda_{j k}<\partial_{r_{k}} \lambda_{i k}<0$, for all $0<k<j<i$.

Proof 1. Direct consequence of Lemma 2.3.

2. We have

$$
\lambda_{i j}= \begin{cases}-\frac{m_{0}}{r_{i}^{3}}, & j=0, \\ -\frac{m_{j}}{r_{i}^{3}}\left(\phi_{1}\left(y_{j}\right)+y_{j} \phi_{1}^{\prime}\left(y_{j}\right)\right), & 0<j<i, \\ \frac{m_{j}}{r_{i}^{3}} x_{j}^{2} \phi_{1}^{\prime}\left(x_{j}\right), & j>i,\end{cases}
$$

and the chain rule gives

$$
\partial_{r_{j}} \lambda_{i j}= \begin{cases}0, & j=0, \\ \frac{\partial y_{j}}{\partial r_{j}} \frac{\partial \lambda_{i j}}{\partial y_{j}}=\frac{1}{r_{i}} \frac{\partial \lambda_{i j}}{\partial y_{j}}<0, & 0<j<i, \\ \frac{\partial x_{j}}{\partial r_{j}} \frac{\partial \lambda_{i j}}{\partial x_{j}}=-\frac{r_{i}}{r_{j}^{2}} \frac{\partial \lambda_{i j}}{\partial x_{j}}<0, & j>i,\end{cases}
$$

by Lemma 2.3 .

3. When $i=j, \lambda_{i 0}=-\frac{m_{0}}{r_{i}^{3}}$ and $\lambda_{i i}=-\frac{m_{i}}{2^{3 / 2} r_{i}^{3}} \zeta_{\ell}$, which are both increasing in $r_{i}$. For $0<j<i$, by Lemma 2.3, we have

$$
\frac{\partial \lambda_{i j}}{\partial r_{i}}=m_{j}\left(\frac{3}{r_{i}^{4}}\left(\phi_{1}\left(y_{j}\right)+y_{j} \phi_{1}^{\prime}\left(y_{j}\right)\right)+\frac{r_{j}}{r_{i}^{5}} \frac{\partial}{\partial y_{j}}\left(\phi_{1}\left(y_{j}\right)+y_{j} \phi_{1}^{\prime}\left(y_{j}\right)\right)\right)>0 .
$$


For $j>i$, we have

$$
\frac{\partial \lambda_{i j}}{\partial r_{i}}=\frac{m_{j}}{r_{j}^{3}} \frac{\partial x_{j}}{\partial r_{i}} \frac{\partial}{\partial x_{j}}\left(\frac{\phi_{1}^{\prime}\left(x_{j}\right)}{x_{j}}\right)=\frac{m_{j}}{r_{j}^{4}} \frac{x_{j} \phi_{1}^{\prime \prime}\left(x_{j}\right)-\phi_{1}^{\prime}\left(x_{j}\right)}{x_{j}^{2}} .
$$

By Lemma 2.3, $\phi_{1}(x)$ is analytic on $(0,1)$ so $\phi_{1}(x)=\sum_{n \geq 0} a_{n} x^{n}$ with $a_{n} \geq 0$. Moreover, $\sum_{k=0}^{\ell-1} e^{i \theta_{k}}=0$ (since it is the sum of the roots of $z^{\ell}-1=0$ ), yielding $a_{1}=\phi_{1}^{\prime}(0)=\sum_{k=0}^{\ell-1} \cos \theta_{k}=0$. Hence,

$$
\begin{aligned}
x \phi_{1}^{\prime \prime}(x)-\phi_{1}^{\prime}(x) & =\sum_{n \geq 2} a_{n} n(n-1) x^{n-1}-\sum_{n \geq 1} a_{n} n x^{n-1} \\
& =\sum_{n \geq 2} a_{n} n(n-2) x^{n-1}>0 .
\end{aligned}
$$

4. Let $k>j>i$ and define $x_{s}=r_{s} / r_{k}$ for $s=i, j$. The derivative according to $r_{k}$ is

$$
\frac{\partial \lambda_{s k}}{\partial r_{k}}=\frac{m_{k}}{r_{s}^{3}} \frac{\partial x_{s}}{\partial r_{k}} \frac{\partial}{\partial x_{s}}\left(x_{s}^{2} \phi_{1}^{\prime}\left(x_{s}\right)\right)=-\frac{m_{k}}{r_{k}^{4}} \frac{x_{s} \phi_{1}^{\prime \prime}\left(x_{s}\right)+2 \phi_{1}^{\prime}\left(x_{s}\right)}{x_{s}}<0 .
$$

Now, because $a_{1}=0,\left(x \phi_{1}^{\prime \prime}(x)+2 \phi_{1}^{\prime}(x)\right) / x$ is strictly positive and increasing. Since $x_{i}<x_{j}$, we deduce that $0>\partial_{r_{k}} \lambda_{i k}>\partial_{r_{k}} \lambda_{j k}$.

5. Let $0<k<j<i$ and $y_{s}=r_{k} / r_{s}$ for $s=i, j$. We have

$$
\frac{\partial \lambda_{s k}}{\partial r_{k}}=-\frac{m_{k}}{r_{s}^{4}}\left(2 \phi_{1}^{\prime}\left(y_{s}\right)+y_{s} \phi_{1}^{\prime \prime}\left(y_{s}\right)\right)<0 .
$$

The function $y \phi_{1}^{\prime \prime}(y)+2 \phi_{1}^{\prime}(y)$ is strictly positive and increasing. Since $y_{j}<y_{i}$, we get $\partial_{r_{k}} \lambda_{i k}<\partial_{r_{k}} \lambda_{j k}<0$.

Corollary 2.5 Let $\Lambda=\left(\Lambda_{1}, \ldots, \Lambda_{n-1}\right) \in \mathbb{R}^{n-1}$ with

$$
\Lambda_{i}(r)=\lambda_{i}(r)-\lambda_{i+1}(r), \quad i=1, \ldots, n-1 .
$$

We have the following properties:

1. $\partial_{r_{i}} \Lambda_{i}>0$ for all $i$.

2. $\partial_{r_{i+1}} \Lambda_{i}<0$ for all $i$.

3. If $j>i+1$, then $\partial_{r_{j}} \Lambda_{i}>0$.

4. If $j<i$, then $\partial_{r_{j}} \Lambda_{i}<0$.

Lemma 2.6 Let $n \in\{2,3,4\}, \ell \in \mathbb{N}_{>0}$ and $\left(m_{0}, m\right) \in \mathbb{R}_{\geq 0} \times \mathbb{R}_{>0}^{n}$. There exists $p \in \mathcal{R}^{n}$, such that the $n \times \ell+1$ spiderweb configuration respects

$$
\lambda_{1}(p)<\cdots<\lambda_{n}(p) .
$$


Furthermore, in the case $n=4$, we may choose $p_{4}$ such that

$$
\lambda_{3}\left(p_{1}, r_{2}, p_{3}, p_{4}\right)<\lambda_{4}\left(p_{1}, r_{2}, p_{3}, p_{4}\right), \quad \forall r_{2} \in\left(p_{1}, p_{2}\right] .
$$

Proof We start with an initial circle located at $p_{1} \in \mathbb{R}_{>0}$. For $n=2$, we have $\lambda_{1}\left(p_{1},+\infty\right)<\lambda_{2}\left(p_{1},+\infty\right)=0$ and this inequality is preserved for $r_{2}=p_{2}<+\infty$ sufficiently large. We can repeat this argument for $n=3$ by adding a third circle from infinity and let $r_{3}$ decrease to $p_{3}<+\infty$ sufficiently large. (Point 3 of Corollary 2.5 guarantees that $\lambda_{1}\left(p_{1}, p_{2}, r_{3}\right)<\lambda_{2}\left(p_{1}, p_{2}, r_{3}\right)$ holds.) We repeat this argument for $n=4$. Moreover, for $p_{4}$ sufficiently large, $\lambda_{4}$ as well as the positive quantity $\lambda_{34}$ of $\lambda_{3}$ can be made small enough to obtain $\lambda_{3}\left(p_{1}, r_{2}, p_{3}, p_{4}\right)<\lambda_{4}\left(p_{1}, r_{2}, p_{3}, p_{4}\right)$ for all $r_{2} \in\left(p_{1}, p_{2}\right]$.

\subsection{Equations for Spiderweb Central Configurations}

Depending of the method we will use, a spiderweb central configuration can be seen as a solution of $\Lambda=0$, as defined in (2.6), or as a zero of the map $f=\left(f_{1}, \ldots, f_{n}\right)$ : $\mathbb{R}^{n} \longrightarrow \mathbb{R}^{n}$ given by

$$
\begin{aligned}
& f_{i}\left(r_{i}, y_{1, i}, \ldots, y_{i-1, i}, x_{i+1, i}, \ldots, x_{n, i}\right) \\
& =\lambda r_{i}-\frac{F_{i}\left(r_{i}, y_{1, i}, \ldots, y_{i-1, i}, x_{i+1, i}, \ldots, x_{n, i}\right)}{m_{i}} \\
& =\lambda r_{i}+\frac{1}{r_{i}^{2}}\left(\frac{m_{i}}{2^{3 / 2}} \zeta_{\ell}+m_{0}+\sum_{j=1}^{i-1} m_{j}\left(\phi_{1}\left(y_{j, i}\right)+y_{j, i} \phi_{1}^{\prime}\left(y_{j, i}\right)\right)\right. \\
& \left.\quad-\sum_{j=i+1}^{n} m_{j} x_{j, i}^{2} \phi_{1}^{\prime}\left(x_{j, i}\right)\right),
\end{aligned}
$$

where we exhibit the dependency in $i$ in (2.3), that is

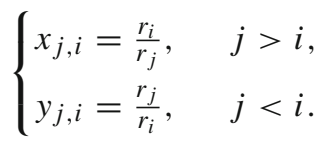

As explained in Sect. 2.1, the common $\lambda$ characterizing a central configuration appearing in (2.7) is a strictly negative fixed constant, independent of $n, \ell$ and of the mass distribution $\left(m_{0}, m\right)$.

\section{Existence of Spiderweb Central Configurations with Arbitrary $n$ and $\ell$}

In this section we give a very short proof of the theorem announced in [2]. This requires introducing the tool of restricted spiderweb central configurations, which will be used also later in the paper. 


\subsection{Restricted Spiderweb Central Configurations}

Theorem 3.1 Let $n \in \mathbb{N}, \ell \in \mathbb{N}_{\geq 2}$ and $\left(m_{0}, m\right) \in \mathbb{R}_{\geq 0} \times \mathbb{R}_{>0}^{n}$. Suppose there exists some radii $r \in \mathcal{R}^{n}$ giving a $n \times \ell+1$ spiderweb central configuration. Then, for any $i \in\{0, \ldots, n\}$, if $m_{n+1}=0$, there exists a unique position

$$
r_{n+1} \in \begin{cases}\left(r_{i}, r_{i+1}\right), & i=0, \ldots, n-1, \\ \left(r_{n},+\infty\right), & i=n,\end{cases}
$$

giving (for $n$ replaced by $n+1)$ a solution of $(2.7)$, i.e. $(n+1) \times \ell+1$ restricted spiderweb central configuration.

Proof By hypothesis, there exists $r \in \mathcal{R}^{n}$ such that the $n \times \ell+1$ spiderweb configuration is central.

Fix $i \in\{0, \ldots, n\}$ and add a $(n+1)^{t h}$ circle of zero mass and of radius

$$
r_{n+1} \in \begin{cases}\left(r_{i}, r_{i+1}\right), & i<n, \\ \left(r_{n},+\infty\right), & i=n\end{cases}
$$

Consider

$$
\lambda_{j}\left(r, r_{n+1}\right)=\frac{F_{j}\left(r, r_{n+1}\right)}{m_{j} r_{j}}, \quad j=1, \ldots, n+1,
$$

which, by (2.5), is perfectly defined when $m_{n+1}=0$.

On the one hand, adding particles of negligible mass bears no effect on the gravitational force felt by the particles on the $n$ initial circle, that is $\lambda_{1}=\ldots=\lambda_{n}=\lambda<0$.

On the other hand, Lemma 2.4 gives the monotonous limits

$$
\lim _{r_{n+1} \searrow r_{i}} \lambda_{n+1}\left(r, r_{n+1}\right)=-\infty, \quad \begin{cases}\lim _{r_{n+1} \nearrow r_{i+1}} \lambda_{n+1}\left(r, r_{n+1}\right)=+\infty, & i<n+1, \\ \lim _{r_{n+1} \rightarrow+\infty} \lambda_{n+1}\left(r, r_{n+1}\right)=0^{-}, & i=n,\end{cases}
$$

Consequently, there is a unique $r_{n+1}$ such that $\lambda_{n+1}=\lambda$.

\subsection{Proof of the Existence of Central Configurations}

Theorem 3.2 Let $n \in \mathbb{N}, \ell \in \mathbb{N}_{\geq 2}$ and $\left(m_{0}, m_{1}\right) \in \mathbb{R}_{\geq 0} \times \mathbb{R}_{>0}$. There exists $r \in \mathcal{R}^{n}$ and masses $m_{1} \gg m_{2} \gg \cdots \gg m_{n}$ giving $a n \times \ell+1$ spiderweb central configuration.

Proof The proof is by induction on $n$. Let $m_{0} \in \mathbb{R}_{\geq 0}$ and $\lambda<0$. If $n=1$, for any $m_{1} \in \mathbb{R}_{>0}$, according to equation (2.7), there exists a unique zero $f\left(r_{1}\right)=f_{1}\left(r_{1}\right)=0$ and the derivative never vanishes according to Lemma 2.3.

Let $n \geq 2$, and suppose that the jacobian $\left|D_{\left(r_{1}, \ldots, r_{n-1}\right)} f\right|$ is invertible for $n-1$ circles with $m_{1} \gg \cdots \gg m_{n-1}$. We place a $n^{t h}$ circle with $m_{n}=0$ and, by theorem 3.1, 
there exists a unique $r(0)=\left(r_{1}, \ldots, r_{n-1}, r_{n}\right) \in \mathcal{R}^{n}$ giving a spiderweb central configuration.

Using the notation $\partial_{k}=\frac{\partial}{\partial r_{k}}$, we have

$\partial_{i} f_{i}$

$$
\begin{aligned}
= & \lambda-\frac{1}{r_{i}^{3}}\left(\frac{m_{i}}{\sqrt{2}} \zeta_{\ell}-2 m_{0}-\sum_{j=1}^{i-1} m_{j}\left(2 \phi_{1}\left(y_{j}\right)+4 y_{j} \phi_{1}^{\prime}\left(y_{j}\right)+y_{j}^{2} \phi_{1}^{\prime \prime}\left(y_{j}\right)\right)\right. \\
& \left.-\sum_{j=i+1}^{n} m_{j} x_{j}^{3} \phi_{1}^{\prime \prime}\left(x_{j}\right)\right), \\
\partial_{j} f_{i}= & \begin{cases}\frac{m_{j}}{r_{i}^{3}}\left(2 \phi_{1}^{\prime}\left(y_{j}\right)+y_{j} \phi_{1}^{\prime \prime}\left(y_{j}\right)\right), & j<i \\
\frac{m_{j} x_{j}^{3}}{r_{i}^{3}}\left(2 \phi_{1}^{\prime}\left(x_{j}\right)+x_{j} \phi_{1}^{\prime \prime}\left(x_{j}\right)\right), & j>i\end{cases}
\end{aligned}
$$

Therefore,

$$
\left|D_{r} f(r(0))\right|=\left|\left(\begin{array}{ccc} 
& & 0 \\
& D_{\left(r_{1}, \ldots, r_{n-1}\right)} f(r(0)) & \vdots \\
& & 0 \\
\hdashline \partial_{1} f_{n}(r(0)) & \ldots & \partial_{n-1} f_{n}(r(0))
\end{array}\right)\right| \neq 0,
$$

because $\partial_{n} f_{n}(r(0))<0$ by Lemma 2.3 and $\left|D_{\left(r_{1}, \ldots, r_{n-1}\right)} f(r(0))\right| \neq 0$ by hypothesis.

For fixed $\left(m_{0}, \ldots, m_{n-1}\right)$, the implicit function theorem yields a neighborhood $V$ of $m_{n}=0$ such that the function $r=r\left(m_{n}\right)$ is a zero of $f$ for all $m_{n} \in V$. So, the condition $m_{n} \ll m_{n-1}$ ensures the existence of the spiderweb central configuration.

\section{Existence and Uniqueness for Circles of Low Density ( $\ell$ Small)}

Recall the map $f$ given in (2.7), whose zeros give a spiderweb central configuration, and its Jacobian matrix $D_{r} f$ given in (3.1). In Theorem 3.2, the existence of a spiderweb central configuration is asserted under the condition $m_{1} \gg \cdots \gg m_{n}$. However, for a system with circles of low density, namely small values of $\ell$, the implicit function theorem may be used to extend the zeros of $f$ for any positive value of the mass $m_{n}$ on the outermost circle. In such cases, iterating the argument allows us to construct a spiderweb central configuration for an arbitrary number $n$ of circles and, moreover, to prove its uniqueness.

Remark 4.1 The proofs of Theorems 4.2 and 4.3 require to show the positivity of some smooth function in some compact interval of $\mathbb{R}$. To avoid fastidious proofs, 
we implement a rigorous method to show the positivity for functions of class $C^{1}$ on $[a, b] \subset \mathbb{R}$.

Let $h \in C^{1}([a, b])$. Plotting the function on $[a, b]$, we see that it is positive. Looking at the plot, we choose a lower bound $\omega_{1}>0$ (for instance $\omega_{1}=\frac{\min _{s \in[a, b]} h(s) \text { ) }}{2}$ ). Let us now choose $\omega_{2}$, for which we can rigorously prove that $\omega_{2}>\max _{s \in[a, b]}\left|h^{\prime}(s)\right|$. Let $p>\omega_{2} / \omega_{1}$. Define a uniform partition by $s_{j}=a+b \times j / p$, for $j=0, \ldots, p$, such that $\bigcup_{j=0}^{p}\left[s_{j}, s_{j+1}\right]=[a, b]$. For any $s \in\left[s_{j}, s_{j+1}\right]$, we have $\left|h(s)-h\left(s_{j+1}\right)\right|<\omega_{2} / p$, in particular $h(s)>h\left(s_{j+1}\right)-\omega_{2} / p>h\left(s_{j+1}\right)-\omega_{1}$.

Thus, it is sufficient to verify rigorously (by means of interval arithmetics, e.g. [1]) that $h\left(s_{j+1}\right)>\omega_{1}$, for $j=0, \ldots, p-1$, to obtain $h(s)>0$ for all $s \in$ $\bigcup_{j=0}^{p}\left[s_{j}, s_{j+1}\right]=[a, b]$. Algorithm 1 implements this method. If it returns an error, then the user should try a smaller $\omega_{1}$.

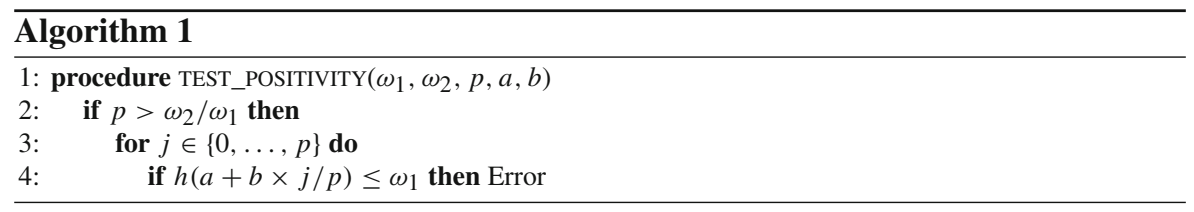

Theorem 4.2 Let $n \in \mathbb{N}, \ell \in\{2, \ldots, 9\}$ and $\left(m_{0}, m\right) \in \mathbb{R}_{\geq 0} \times \mathbb{R}_{>0}^{n}$. For a fix $\lambda$, there exists a unique $r \in \mathcal{R}^{n}$ such that the $n \times \ell+1$ spiderweb configuration is central.

Proof For $n=1$, we have a regular $\ell$-gon with a central mass. The equation (2.7) shows the existence of a unique $r_{1} \in \mathbb{R}_{>0}$ such that the configuration is central.

Suppose that for $n-1$ circles, with $\left(m_{1}, \ldots, m_{n-1}\right) \in \mathbb{R}_{>0}^{n-1}$, there exists a unique $\left(r_{1}, \ldots, r_{n-1}\right) \in \mathcal{R}^{n-1}$ such that the $(n-1) \times \ell+1$ mass-particles form a spiderweb central configuration for $\lambda$. By Theorem 3.1, there exists a unique $r(0)=\left(r_{1}, \ldots, r_{n-1}, r_{n}\right) \in \mathcal{R}^{n}$ such that the $n \times \ell+1$ spiderweb restricted configuration with $m_{n}=0$ is central for this $\lambda$. We want to extend this solution for all positive values of $m_{n}$.

The structure of the proof is the following:

Claim 1: The jacobian matrix $D_{r} f \in M_{n}(\mathbb{R})$, whose entries are given by (3.1), is invertible for all $r \in \mathcal{R}^{n}$ and $m_{n} \in \mathbb{R}_{\geq 0}$.

Claim 2: All the radii remain bounded for all $m_{n} \in \mathbb{R}_{\geq 0}$.

Claim 3: All the radii remain distinct for all $m_{n} \in \mathbb{R}_{\geq 0}$.

The first claim allows using the implicit function theorem to obtain a function $r\left(m_{n}\right)$ such that $f\left(r\left(m_{n}\right), m_{n}\right)=0$. Claims 2 and 3 allow concluding that $r\left(m_{n}\right)$ can be uniquely extended for any value of $m_{n} \in \mathbb{R}_{\geq 0}$, yielding its local uniqueness. The global uniqueness follows from the following argument: suppose there is an other function $\psi\left(m_{n}\right)$ such that $f\left(\psi\left(m_{n}\right), m_{n}\right)=0$, then it can be extended on $\mathbb{R}_{\geq 0}$. In particular, $\psi(0)=r(0)$ because $r(0)$ is unique. Hence, $\psi\left(m_{n}\right)=r\left(m_{n}\right)$ for every $m_{n} \in \mathbb{R}_{\geq 0}$. 
Claim 1: Recall that a sufficient criterion for a matrix to be invertible is to be strictly diagonally dominant. ${ }^{1}$ We know that $\zeta_{\ell}$ is strictly positive and, by Lemma 2.3, that $\partial_{i} f_{i}<0$ and $\partial_{j} f_{i}>0$ for $j \neq i$. Hence, we must show

$$
-\partial_{i} f_{i}-\sum_{\substack{j=1 \\ j \neq i}}^{n} \partial_{j} f_{i}>0, \quad i=1, \ldots, n
$$

Now, if we rewrite $f_{i}$ in terms of $x_{j}$ and $\phi$ defined respectively in (2.3) and (2.4)

$$
\begin{aligned}
& -\partial_{i} f_{i}-\sum_{\substack{j=1 \\
j \neq i}}^{n} \partial_{j} f_{i} \\
& =-\lambda+\frac{1}{r_{i}^{3}}\left(\frac{m_{i}}{\sqrt{2}} \zeta_{\ell}+2 m_{0}+\sum_{\substack{j=1 \\
j \neq i}}^{n} m_{j} x_{j}^{3}\left(\phi_{1}^{\prime \prime}\left(x_{j}\right)\left(1-x_{j}\right)-2 \phi_{1}^{\prime}\left(x_{j}\right)\right)\right) .
\end{aligned}
$$

But,

$$
\begin{aligned}
\phi_{1}^{\prime}\left(x_{j}\right) & =\frac{d}{d x_{j}}\left(\sum_{k=0}^{\ell-1} \frac{1}{\left(1+x_{j}^{2}-2 x_{j} \cos \theta_{k}\right)^{1 / 2}}\right) \\
& =-\sum_{k=0}^{\ell-1} \frac{x_{j}-\cos \theta_{k}}{\left(1+x_{j}^{2}-2 x_{j} \cos \theta_{k}\right)^{3 / 2}}, \\
\phi_{1}^{\prime \prime}\left(x_{j}\right) & =\frac{d}{d x_{j}}\left(-\sum_{k=0}^{\ell-1} \frac{x_{j}-\cos \theta_{k}}{\left(1+x_{j}^{2}-2 x_{j} \cos \theta_{k}\right)^{3 / 2}}\right) \\
& =-\sum_{k=0}^{\ell-1} \frac{1+x_{j}^{2}-2 x_{j} \cos \theta_{k}-3\left(x_{j}-\cos \theta_{k}\right)^{2}}{\left(1+x_{j}^{2}-2 x_{j} \cos \theta_{k}\right)^{5 / 2}},
\end{aligned}
$$

whence

$$
\begin{aligned}
& \phi_{1}^{\prime \prime}\left(x_{j}\right)\left(1-x_{j}\right)-2 \phi_{1}^{\prime}\left(x_{j}\right) \\
& =\sum_{k=0}^{\ell-1} \frac{\left(1-\cos \theta_{k}\right)\left(2 x_{j}^{2}+x_{j}\left(3-\cos \theta_{k}\right)-1-3 \cos \theta_{k}\right)}{\left(1+x_{j}^{2}-2 x_{j} \cos \theta_{k}\right)^{5 / 2}} .
\end{aligned}
$$

$\overline{1}$ i.e. $\left|M_{i i}\right|>\left|\sum_{j \neq i} M_{i j}\right|$ for $i=1, \ldots, n$ and $M \in M_{n}(\mathbb{R})$. 
Noticing that the term for $k=0$ is zero, we find

$$
-\partial_{i} f_{i}-\sum_{\substack{j=1 \\ j \neq i}}^{n} \partial_{j} f_{i}=-\lambda+\frac{1}{r_{i}^{3}}\left(\frac{m_{i}}{\sqrt{2}} \zeta_{\ell}+2 m_{0}+\sum_{\substack{j=1 \\ j \neq i}}^{n} m_{j} x_{j}^{3} h_{\ell}\left(x_{j}\right)\right),
$$

with

$$
h_{\ell}\left(x_{j}\right)=\sum_{k=1}^{\ell-1} \frac{\left(1-\cos \theta_{k}\right)\left(2 x_{j}^{2}+x_{j}\left(3-\cos \theta_{k}\right)-1-3 \cos \theta_{k}\right)}{\left(1+x_{j}^{2}-2 x_{j} \cos \theta_{k}\right)^{5 / 2}} .
$$

Since $\lambda<0$ and $\zeta_{\ell}>0$, the expression given in (4.1) is strictly positive if $h_{\ell}\left(x_{j}\right)$ is positive, where the sign of the latter depends on the sign of the polynomial $2 x^{2}+$ $x\left(3-\cos \theta_{k}\right)-1-3 \cos \theta_{k}$.

It is sufficient to show that the sign of $h_{\ell}\left(x_{j}\right)$ is strictly positive in the case $x_{j} \in$ $(0,1)$, that is when $j>i$. Indeed, for $x>1$, we have

$$
2 x^{2}+x\left(3-\cos \theta_{k}\right)-1-3 \cos \theta_{k}>2\left(x^{2}+x\right)-4>0 .
$$

Cases $\ell=2,3,4$

For $\ell=2,3$, we have

$$
h_{\ell}\left(x_{j}\right)= \begin{cases}\frac{4}{\left(1+x_{j}\right)^{3}}, & \ell=2, \\ \frac{3\left(\frac{1}{2}+\frac{7}{2} x+2 x^{2}\right)}{\left(1+x_{j}+x_{j}^{2}\right)^{5 / 2}}, & \ell=3,\end{cases}
$$

which are clearly strictly positive function for all $x_{j} \in(0,1)$.

For $\ell=4$, we have

$$
h_{4}\left(x_{j}\right)=\frac{4}{\left(1+x_{j}\right)^{3}}+2 \frac{2 x_{j}^{2}+3 x_{j}-1}{\left(1+x_{j}^{2}\right)^{5 / 2}} .
$$

It is sufficient to show that $v(x)=(1+x)^{3} h_{4}(x)$ is strictly positive for $x \in(0,1)$. The derivative is $v^{\prime}(x)=6 x(1+x)^{2}\left(7-3 x^{2}\right)\left(1+x^{2}\right)^{-7 / 2}$ whose only positive root is strictly greater than 1 . Since $v(0)>0$, it follows that $v(x)>0$ for all $x \in(0,1)$.

Cases $\ell=5, \ldots, 9$

For each value of $\ell$, we apply Algorithm 1 to $h_{\ell} \in C^{\infty}([0,1])$.

Therefore, we have established that $-\partial_{i} f_{i}-\sum_{\substack{j=1 \\ j \neq i}}^{n} \partial_{j} f_{i}$ is a sum of positive terms for $\ell=2, \ldots, 9$, thus proving the first claim.

By the implicit function theorem, we conclude to the existence of a function $r=$ $r\left(m_{n}\right)$ defined on a neighborhood $V$ of $m_{n}=0$, such that $f\left(r\left(m_{n}\right), m_{n}\right)=0$ for all $m_{n} \in V$. 
Claim 2: Let us suppose that $\sup V<+\infty$. Let $\left\{a_{k}\right\}_{k \geq 1}$ be a sequence in $V$ such that $\lim _{k \rightarrow+\infty} a_{k}=\sup V$ and let us suppose that $\lim _{k \rightarrow+\infty} r_{i}\left(a_{k}\right)=+\infty$ for some index $i$.

If $i=1$, then (2.7) gives the contradiction since $0=f_{1}\left(r\left(a_{k}\right), a_{k}\right) \rightarrow-\infty$ as $k \rightarrow+\infty$.

Otherwise, looking at $f_{i}\left(r\left(a_{k}\right), a_{k}\right)=0$, since $\lim _{k \rightarrow+\infty} \lambda r_{i}\left(a_{k}\right)=-\infty$ we must have

$$
\lim _{k \rightarrow+\infty} \frac{1}{r_{i}\left(a_{k}\right)^{2}}\left(\phi_{1}\left(y_{i-1, i}\left(a_{k}\right)\right)+y_{i-1, i}\left(a_{k}\right) \phi_{1}^{\prime}\left(y_{i-1, i}\left(a_{k}\right)\right)\right)=+\infty,
$$

which can only occur if $\phi_{1}\left(y_{i-1, i}\left(a_{k}\right)\right) \rightarrow+\infty$. The latter occurs when $y_{i-1, i}\left(a_{k}\right)=$ $r_{i-1}\left(a_{k}\right) / r_{i}\left(a_{k}\right) \rightarrow 1$ as $k \rightarrow+\infty$, i.e. $\lim _{k \rightarrow+\infty} r_{i-1}\left(a_{k}\right)=+\infty$. Looking at $f_{i-1}$ in (2.7), we iterate the reasoning if $i>2$ to obtain $\lim _{k \rightarrow+\infty} r_{i-2}\left(a_{k}\right)=+\infty$, etc. We finally conclude that $\lim _{k \rightarrow+\infty} r_{1}\left(a_{k}\right)=+\infty$ yielding once again the contradiction $0=f_{1}\left(r\left(a_{k}\right), a_{k}\right) \rightarrow-\infty$ as $k \rightarrow+\infty$.

Claim 3: Let $V$ and $\left\{a_{k}\right\}_{k \geq 1}$ as previously defined.

Suppose that for an index $i$ we have $\lim _{k \rightarrow+\infty} x_{i+1, i}\left(a_{k}\right)=r_{i}\left(a_{k}\right) / r_{i+1}\left(a_{k}\right)=1$.

If $i=1$, then we have the contradiction because of the infinite negative term coming from $\lim _{k \rightarrow \infty} \phi_{1}^{\prime}\left(x_{2,1}\left(a_{k}\right)\right)$, in the equation for $f_{1}$ in (2.7), while the positive terms remain bounded.

Otherwise for $i>1$, due to the singularity at 1 of $\phi_{1}$, preserving the equality $f_{i}\left(r\left(a_{k}\right), a_{k}\right)=0$ implies $\lim _{k \rightarrow+\infty} y_{i-1, i}=r_{i-1}\left(a_{k}\right) / r_{i}\left(a_{k}\right)=1$. But, $y_{i-1, i}=$ $x_{i, i-1}$ so looking at $f_{i-1}$ imposes $\lim _{k \rightarrow+\infty} y_{i-2, i-1}=r_{i-2}\left(a_{k}\right) / r_{i-1}\left(a_{k}\right)=1$ provided $i>2$. Iterating this argument, we find $\lim _{k \rightarrow+\infty} r_{1}\left(a_{k}\right) / r_{2}\left(a_{k}\right)=1$, so again we have the contradiction $0=f_{1}\left(r\left(a_{k}\right), a_{k}\right) \rightarrow-\infty$.

Theorem 4.3 Let $n \in \mathbb{N}$ and $\left(m_{0}, m\right) \in \mathbb{R}_{\geq 0} \times \mathbb{R}_{>0}^{n}$. If $m_{1} \geq \ldots \geq m_{n}$, then the $n \times \ell+1$ spiderweb central configuration exists and is unique for a fix $\lambda$ in the following cases:

1. $\ell=10$ and $n \leq 17$;

2. $\ell=11$ and $n \leq 9$;

3. $\ell=12$ and $n \leq 6$;

4. $\ell=13$ and $n \leq 5$;

5. $\ell=14,15$ and $n \leq 4$;

6. $\ell=16,17,18$ and $n \leq 3$.

Proof Identically to the preceding proof, and noting that Claims 2 and 3 are independent of $\ell$ it remains to establish Claim 1 in this context.

As shown on Fig. 2, $h_{\ell}\left(x_{j}\right)$ is now negative for some $x_{j} \in(0,1)$. Considering $m_{1} \geq \ldots \geq m_{n}$ and equation (4.1), we have the inequality

$$
\frac{m_{i}}{\sqrt{2}} \zeta_{\ell}+2 m_{0}+\sum_{\substack{j=1 \\ j \neq i}}^{n} m_{j} x_{j}^{3} h_{\ell}\left(x_{j}\right) \geq 2 m_{0}+\frac{m_{i}}{\sqrt{2}}\left(\zeta_{\ell}+\sqrt{2}(n-1) \min _{x \in[0,1]} h_{\ell}(x)\right) .
$$




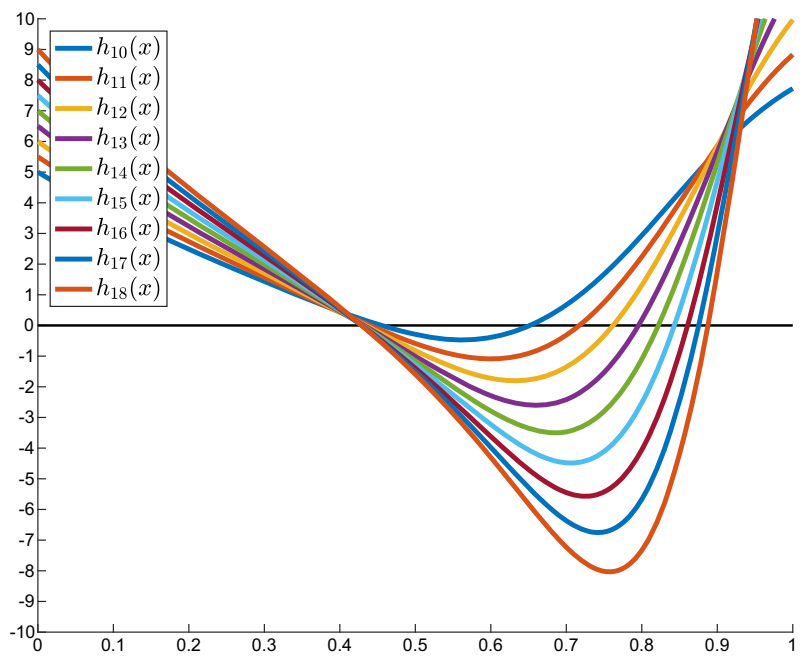

Fig. 2 Graph of $h_{\ell}(x)$ in the interval $[0,1]$ for $\ell=10, \ldots, 18$

For each value of $\ell$, we use Algorithm 1 on $h_{\ell}-\omega(\ell) \in C^{\infty}([0,1])$ with $\omega(\ell)<\min _{x \in[0,1]} h_{\ell}<0$. $^{2}$ It is now easy to check that $n$ cannot be greater than $17,9,6,5,4,4,3,3,3$ for $\ell=10, \ldots, 18$ respectively, to obtain $\zeta_{\ell}+\sqrt{2}(n-$ 1) $\min _{x \in[0,1]} h_{\ell}(x)>0$.

\section{Constructive Proof of Existence for $n \in\{3,4\}$ and Arbitrary $\ell$}

The proof is a completion of Saari's proof given in [11] for the existence of spiderweb central configurations when $n \in\{3,4\}$. It makes an essential use of all properties proved in Lemma 2.4 and Corollary 2.5.

Theorem 5.1 Let $n \in \mathbb{N}, \ell \in \mathbb{N}_{\geq 2}$ and $\left(m_{0}, m\right) \in \mathbb{R}_{\geq 0} \times \mathbb{R}_{>0}^{n}$. If $n \leq 4$, then there exists a $n \times \ell+1$ spiderweb central configuration.

Furthermore, in the case $n=2$, it is the unique such configuration for a fix $\lambda$. (The result for $n=2$ is already in [7]).

2 E.g.

$$
\omega(\ell)= \begin{cases}-0.48, & \ell=10 \\ -1.1, & \ell=11 \\ -1.82, & \ell=12 \\ -2.61, & \ell=13 \\ -4, & \ell=14 \\ -4.5, & \ell=15 \\ -5.6, & \ell=16 \\ -7, & \ell=17 \\ -8.2, & \ell=18\end{cases}
$$


Proof The proof consists in three parts: Part A, Part B and Part C. Part A shows that for a number of $n \in\{2,3,4\}$ circles, there exists radii for two consecutive ones, say $i$-th and $(i+1)$-th, such that $\Lambda_{i}=0$. This implies the case $n=2$ of the theorem. By means of Part A, we prove the cases $n=3$ and 4 of the theorem in Part B and Part C respectively.

\section{Part A}

Let $n \in\{2,3,4\}$ be the number of circles, and fix $i \in\{1,2,3\}$. By Lemma 2.6, there exists an initial position $p \in \mathcal{R}^{n}$ for the $n$ circles such that $\Lambda_{i}(p)<0$. Moreover, Corollary 2.5 implies the monotonous limit $\lim _{r_{i+1} \searrow p_{i}} \Lambda_{i}\left(r_{i+1}\right)=+\infty$. Thus, the function $\Lambda_{i}$ has a unique zero. Taking $n=2$ gives the unique spiderweb central configuration.

\section{Part B}

Let $n=3$. Lemma 2.6 gives us an initial position such that the three circles satisfy $\lambda_{1}(p)<\lambda_{2}(p)<\lambda_{3}(p)<0$. We drop the dependency on $p_{1}$ as we keep $p_{1}$ fixed.

Now, from Part A we know that we can bring the third circle closer to the second one to a unique $r_{3}^{*}$ such that

$$
\lambda_{1}\left(p_{2}, r_{3}^{*}\right)<\lambda_{2}\left(p_{2}, r_{3}^{*}\right)=\lambda_{3}\left(p_{2}, r_{3}^{*}\right)
$$

The first inequality remains true because of Point 3 of Corollary 2.5. Point 2 of the same corollary gives $\partial_{r_{3}} \Lambda_{2}<0$ for all $\left(r_{2}, r_{3}\right)$, so, by the implicit function theorem, there is a function $r_{3}=r_{3}\left(r_{2}\right)$, defined on a neighborhood $V$ of $r_{2}=p_{2}$, such that

$$
r_{3}^{*}=r_{3}\left(p_{2}\right) \quad \text { and } \quad \Lambda_{2}\left(r_{2}, r_{3}\left(r_{2}\right)\right)=0, \quad \forall r_{2} \in V
$$

Since for any $r_{2} \in\left(p_{1}, p_{2}\right]$ Part A holds, $r_{3}$ as a function of $r_{2}$ is unique and can be extended for all $r_{2} \in\left(p_{1}, p_{2}\right]$.

Moreover, the function $r_{3}\left(r_{2}\right)$ is strictly increasing since $r_{3}^{\prime}\left(r_{2}\right)=-\frac{\partial_{r_{2}} \Lambda_{2}}{\partial_{r_{3}} \Lambda_{2}}>0$. This implies that the $\operatorname{limit}_{\lim _{r_{2}} \searrow p_{1}} \lambda_{1}\left(r_{2}, r_{3}\left(r_{2}\right)\right)=+\infty$ is monotonous. Meanwhile, $\lambda_{3}<0$ because the $\lambda$ of the outermost circle is always negative.

Thus, by the intermediate value theorem, there exists a $r_{2}^{\text {sol }} \in\left(p_{1}, p_{2}\right)$ such that

$$
\lambda_{1}\left(r_{2}^{s o l}, r_{3}\left(r_{2}^{s o l}\right)\right)=\lambda_{2}\left(r_{2}^{s o l}, r_{3}\left(r_{2}^{s o l}\right)\right)=\lambda_{3}\left(r_{2}^{s o l}, r_{3}\left(r_{2}^{s o l}\right)\right) .
$$

\section{Part C}

Let $n=4$. Once again, by Lemma 2.6 there is an initial position satisfying $\lambda_{1}(p)<$ $\lambda_{2}(p)<\lambda_{3}(p)<\lambda_{4}(p)<0$. Also, we keep $p_{1}$ fixed so that the value of $\lambda_{i}$ do not depend on $p_{1}$ anymore. that

By Part A, we may bring the fourth circle closer to the third to a unique $r_{4}^{*}$ such

$$
\lambda_{1}\left(p_{2}, p_{3}, r_{4}^{*}\right)<\lambda_{2}\left(p_{2}, p_{3}, r_{4}^{*}\right)<\lambda_{3}\left(p_{2}, p_{3}, r_{4}^{*}\right)=\lambda_{4}\left(p_{2}, p_{3}, r_{4}^{*}\right)
$$


Since we have the inequality $\partial_{r_{4}} \Lambda_{3}<0$ for all $\left(r_{2}, r_{3}, r_{4}\right)$, the implicit function theorem gives the existence of a function $r_{4}=z\left(r_{2}, r_{3}\right)$, defined on a neighborhood $V$ of $\left(r_{2}, r_{3}\right)=\left(p_{2}, p_{3}\right)$, such that

$$
z\left(p_{2}, p_{3}\right)=r_{4}^{*} \text { and } \Lambda_{3}\left(r_{2}, r_{3}, z\left(r_{2}, r_{3}\right)\right)=0, \quad \forall\left(r_{2}, r_{3}\right) \in V
$$

However, we know that $\Lambda_{3}\left(r_{2}, r_{3}, p_{4}\right)<0$ for all $\left(r_{2}, r_{3}\right) \in\left(p_{1}, p_{2}\right] \times\left(r_{2}, p_{3}\right]$. So, we can use Part A for each pair of values $\left(r_{2}, r_{3}\right)$. Thus, the function $z\left(r_{2}, r_{3}\right)$ is unique and can be uniquely extended for all $\left(r_{2}, r_{3}\right) \in\left(p_{1}, p_{2}\right] \times\left(r_{2}, p_{3}\right]$.

Moreover, the fact that $\frac{d}{d r_{2}} z\left(r_{2}, p_{3}\right)$ is strictly negative implies that $\Lambda_{1}$ is monotonously increasing in $r_{2}$ to $+\infty$.

Additionally, on the surface $r_{4}=z\left(r_{2}, r_{3}\right)$, we have

$$
\frac{d}{d r_{2}} \Lambda_{2}=\frac{d}{d r_{2}}\left(\lambda_{2}-\lambda_{3}\right)=\frac{d}{d r_{2}}\left(\lambda_{2}-\lambda_{4}\right)>0
$$

Hence, we have $\Lambda_{1}\left(p_{2}, p_{3}, z\left(p_{2}, p_{3}\right)\right), \Lambda_{2}\left(p_{2}, p_{3}, z\left(p_{2}, p_{3}\right)<0\right.$, so there exists a unique $\hat{r}_{2} \in\left(p_{1}, p_{2}\right)$, such that we have the inequality

$$
\lambda_{1}(v, z(v))=\lambda_{2}(v, z(v))<\lambda_{3}(v, z(v))=\lambda_{4}(v, z(v))
$$

where $v=\left(\hat{r}_{2}, p_{3}\right)$.

The point $\left(\hat{r}_{2}, p_{3}\right)$ is then a zero of the function $\mathcal{L}\left(r_{2}, r_{3}, r_{4}\right)=\left(\Lambda_{1}, \Lambda_{3}\right)$ whose jacobian with respect to $\left(r_{3}, r_{4}\right)$ is

$$
\left|D_{\left(r_{3}, r_{4}\right)} \mathcal{L}\right|=\left|\left(\begin{array}{cc}
\partial_{r_{3}} \Lambda_{1} & \partial_{r_{4}} \Lambda_{1} \\
\partial_{r_{3}} \Lambda_{3} & \partial_{r_{4}} \Lambda_{3}
\end{array}\right)\right|<0
$$

By the implicit function theorem, we have the existence of a function $\left(r_{3}, r_{4}\right)=$ $\left(r_{3}\left(r_{2}\right), r_{4}\left(r_{2}\right)\right)=\psi\left(r_{2}\right)$, defined on a neighborhood $V^{\prime}$ of $r_{2}=\hat{r}_{2}$, such that

$$
\psi\left(\hat{r}_{2}\right)=\left(p_{3}, z\left(\hat{r}_{2}, p_{3}\right)\right) \text { and } \mathcal{L}\left(r_{2}, \psi\left(r_{2}\right)\right)=0, \quad \forall r_{2} \in V^{\prime}
$$

A quick look at the signs in

$$
\psi^{\prime}\left(r_{2}\right)=\left(\begin{array}{l}
r_{3}^{\prime}\left(r_{2}\right) \\
r_{4}^{\prime}\left(r_{2}\right)
\end{array}\right)=\frac{-1}{\left|D_{\left(r_{3}, r_{4}\right)} \mathcal{L}\right|}\left(\begin{array}{cc}
\partial_{r_{4}} \Lambda_{3} & -\partial_{r_{4}} \Lambda_{1} \\
-\partial_{r_{3}} \Lambda_{3} & \partial_{r_{3}} \Lambda_{1}
\end{array}\right)\left(\begin{array}{c}
\partial_{r_{2}} \Lambda_{1} \\
\partial_{r_{2}} \Lambda_{3}
\end{array}\right)
$$

shows that $r_{3}^{\prime}\left(r_{2}\right)>0$. Whence, for any $r_{2} \in V^{\prime} \cap\left(p_{1}, \hat{r}_{2}\right]$, we get $r_{3}\left(r_{2}\right) \in\left(r_{2}, p_{3}\right]$. Thus, $\left(r_{2}, \psi\left(r_{2}\right)\right)$ is contained within the surface given by $r_{4}=z\left(r_{2}, r_{3}\right)$ for $\left(r_{2}, r_{3}\right) \in$ $\left(p_{1}, p_{2}\right] \times\left(r_{2}, p_{3}\right]$, and this surface is included in $\mathcal{R}^{3}$ by construction. Hence, provided inf $V^{\prime}>p_{1}$, the radius of the third and fourth circles, given by $\psi\left(r_{2}\right)$, cannot tend to the same limit as $r_{2} \rightarrow \inf V^{\prime}$.

Since the sign of the Jacobian is always strictly negative, the function $\psi\left(r_{2}\right)$ may be uniquely extended for all $r_{2} \in\left(p_{1}, p_{2}\right]$. Notice that $\psi$ is unique since $\hat{r}_{2}$ is unique. 
On the one hand, we have seen that $\lambda_{1}\left(\hat{r}_{2}, \psi\left(\hat{r}_{2}\right)\right)=\lambda_{2}\left(\hat{r}_{2}, \psi\left(\hat{r}_{2}\right)\right)<\lambda_{3}\left(\hat{r}_{2}, \psi\left(\hat{r}_{2}\right)\right)$ $=\lambda_{4}\left(\hat{r}_{2}, \psi\left(\hat{r}_{2}\right)\right)$. On the other hand, we always have the $\operatorname{limit}_{\lim _{2} \searrow p_{1}} \lambda_{1}\left(r_{2}, \psi\left(r_{2}\right)\right)=$ $+\infty$ while $\lambda_{4}$ remains strictly negative. Consequently, the intermediate value theorem implies the existence of $r_{2}^{\text {sol }} \in\left(p_{i}, p_{2}\right)$ such that

$$
\lambda_{1}\left(r_{2}^{s o l}, \psi\left(r_{2}^{s o l}\right)\right)=\lambda_{2}\left(r_{2}^{s o l}, \psi\left(r_{2}^{s o l}\right)\right)=\lambda_{3}\left(r_{2}^{s o l}, \psi\left(r_{2}^{s o l}\right)\right)=\lambda_{4}\left(r_{2}^{s o l}, \psi\left(r_{2}^{s o l}\right)\right)
$$

Remark 5.2 The proof requires multiple uses of the implicit function theorem and, in each case, it was easy to show that the corresponding Jacobian had a fixed sign for all values of the $r_{i}$. Going to $n>4$, there seems no easier way than lengthy calculations for each particular value of $n$ to check the hypotheses of the implicit function theorem each time it is necessary to extend a solution by varying the $r_{i}$.

\section{Computer-Assisted Proof}

Once again, we consider the map $f$ defined in Section 2.7, that is

$$
f_{i}(r)=\lambda r_{i}+\frac{m_{i}}{2^{3 / 2} r_{i}^{2}} \zeta_{\ell}+\frac{m_{0}}{r_{i}^{2}}+\sum_{\substack{j=1 \\ j \neq i}}^{n} \sum_{k=0}^{\ell-1} \frac{m_{j}\left(r_{i}-r_{j} \cos \theta_{k}\right)}{\left(r_{i}^{2}+r_{j}^{2}-2 r_{i} r_{j} \cos \theta_{k}\right)^{3 / 2}}, \quad i=1, \ldots, n
$$

Let $A: \mathbb{R}^{n} \rightarrow \mathbb{R}^{n}$ be a linear operator and define $T: U \longrightarrow X$ by $T(x):=$ $x-A f(x)$ where $U$ is an open set of $\mathbb{R}^{n}$.

Knowing an approximate zero of $f$, the radii polynomial approach gives bounds so that we may find a ball, centered at this approximation, on which $T$ is a contraction to which we can apply the Banach fixed point theorem and $A$ is non singular. Hence, it allows proving the existence and uniqueness of a true solution $\tilde{r} \in \mathbb{R}^{n}$ lying in this ball.

Due to the singularities in the equation (1.1) we must be careful in our numerical approach. We consider the local version in finite dimension of the radii polynomial approach established by Lessard, that is we introduce an upper bound $\rho_{*}$ for the radius of the ball in order to remain away from any singularities.

Theorem 6.1 (Radii polynomial [4]) Let $U$ an open set of $\mathbb{R}^{n}$ and $f: U \rightarrow \mathbb{R}^{n} a$ map of class $C^{1}$. Let $\bar{r} \in U$ and $A \in M_{n}(\mathbb{R})$. Let $\rho_{*}>0$ such that $\overline{B_{\rho_{*}}(\bar{r})} \subset U$. Let $Y_{0}, Z_{0} \in \mathbb{R}$, and $Z_{2}:\left(0, \rho_{*}\right] \rightarrow[0, \infty)$ satisfying

$$
\begin{aligned}
\|A f(\bar{r})\| & \leq Y_{0}, \\
\left\|I d-A D_{r} f(\bar{r})\right\| & \leq Z_{0}, \\
\left\|A\left[D_{r} f(c)-D_{r} f(\bar{r})\right]\right\| & \leq Z_{2}(\rho) \rho, \quad \forall c \in \overline{B_{\rho}(\bar{r})} \text { and } \rho \in\left(0, \rho_{*}\right] .
\end{aligned}
$$


Define the radii polynomial by

$$
p(\rho):=Z_{2}(\rho) \rho^{2}-\left(1-Z_{0}\right) \rho+Y_{0} .
$$

If there exists $\rho_{0} \in\left(0, \rho_{*}\right]$ such that $p\left(\rho_{0}\right)<0$, then $A$ is invertible and there exists a unique $\tilde{r} \in \overline{B_{\rho_{0}}(\bar{r})}$ satisfying $f(\tilde{r})=0$.

Remark 6.2 The choice of $\rho_{*}$ is quite arbitrary. We make an intial heuristic choice and check a posteriori that if there exists $\rho_{0}$ such that $p\left(\rho_{0}\right)<0$ then $\rho_{0}<\rho_{*}$. Otherwise, we must increase the value of $\rho_{*}$.

Remark 6.3 According to Newton's method, the operator $A$ is taken to be the numerical inverse of $D_{r} f(\bar{r})$ where $\bar{r}$ satisfies $f(\bar{r}) \approx 0$ and is found by Newton's method.

Remark 6.4 To rigorously compute the bounds, we use techniques of interval arithmetic (e.g. [1]).

\subsection{Computation of the Bounds}

The bounds $Y_{0}$ and $Z_{0}$ can be found immediately from the theorem, knowing that the Jacobian matrix $D_{r} f$ is given by

$$
\partial_{j} f_{i}= \begin{cases}\lambda-\frac{m_{i}}{r_{i}^{3} \sqrt{2}} \zeta_{\ell}-\frac{2 m_{0}}{r_{i}^{3}}-\sum_{\substack{j=1 \\ j \neq i}}^{n} \frac{m_{j}}{2} \sum_{k=0}^{\ell-1} \frac{4 r_{i}^{2}+r_{j}^{2}-8 r_{i} r_{j} \cos \theta_{k}+3 r_{j}^{2} \cos 2 \theta_{k}}{\left(r_{i}^{2}+r_{j}^{2}-2 r_{i} r_{j} \cos \theta_{k}\right)^{5 / 2}}, & j=i, \\ -\frac{m_{j}}{2} \sum_{k=0}^{\ell-1} \frac{-4\left(r_{i}^{2}+r_{j}^{2}\right) \cos \theta_{k}+r_{i} r_{j}\left(7+\cos 2 \theta_{k}\right)}{\left(r_{i}^{2}+r_{j}^{2}-2 r_{i} r_{j} \cos \theta_{k}\right)^{5 / 2}}, & j \neq i .\end{cases}
$$

For $f \in C^{2}$, it is possible to obtain a general expression for the $Z_{2}$ bound in the infinity norm.

Lemma 6.5 The following equality holds

$$
Z_{2}=\sup _{b \in \overline{B_{\rho_{*}}(\bar{x})}}\left(\max _{1 \leq i \leq n} \sum_{1 \leq k, m \leq n}\left|\sum_{1 \leq j \leq n} A_{i j} \partial_{k m}^{2} f_{j}(b)\right|\right)
$$

Proof Let $c \in \overline{B_{\rho}(\bar{r})}$ for $\rho \in\left(0, \rho_{*}\right)$. By the mean value theorem on the segment $[\bar{x}, c]$ for the function $D_{x} f$ from $\mathbb{R}^{n}$ to $\mathbb{R}^{n^{2}}$ there exists $\xi \in[0,1]$ such that $b=$ $\bar{x}+\xi(c-\bar{x}) \in \overline{B_{\rho_{*}}(\bar{x})}$ and for all $j, m$

$$
\sum_{1 \leq k \leq n} \partial_{k m}^{2} f_{j}(b)(c-\bar{x})_{k}=\partial_{m} f_{j}(c)-\partial_{m} f_{j}(\bar{x}) .
$$


Thus,

$$
\begin{aligned}
\left(A\left[D_{x} f(c)-D_{x} f(\bar{x})\right]\right)_{i m} & =\sum_{1 \leq j \leq n} A_{i j}\left[\partial_{m} f_{j}(c)-\partial_{m} f_{j}(\bar{x})\right] \\
& =\sum_{1 \leq j \leq n} \sum_{1 \leq k \leq n} A_{i j} \partial_{k m}^{2} f_{j}(b)(c-\bar{x})_{k} .
\end{aligned}
$$

Taking the infinity norm,

$$
\begin{aligned}
\left\|A\left[D_{x} f(c)-D_{x} f(\bar{x})\right]\right\|_{\infty} & =\max _{1 \leq i \leq n} \sum_{1 \leq m \leq n}\left|\left(A\left[D_{x} f(c)-D_{x} f(\bar{x})\right]\right)_{i m}\right| \\
& =\max _{1 \leq i \leq n} \sum_{1 \leq m \leq n}\left|\sum_{1 \leq j \leq n} \sum_{1 \leq k \leq n} A_{i j} \partial_{k m}^{2} f_{j}(b)(c-\bar{x})_{k}\right| \\
& \leq \max _{1 \leq i \leq n} \sum_{1 \leq m \leq n} \sum_{1 \leq k \leq n}\left|\sum_{1 \leq j \leq n} A_{i j} \partial_{k m}^{2} f_{j}(b)(c-\bar{x})_{k}\right| \\
& =\max _{1 \leq i \leq n} \sum_{1 \leq k, m \leq n}\left|(c-\bar{x})_{k}\right|\left|\sum_{1 \leq j \leq n} A_{i j} \partial_{k m}^{2} f_{j}(b)\right| \\
& \leq\left(\max _{1 \leq i \leq n} \sum_{1 \leq k, m \leq n}\left|\sum_{1 \leq j \leq n} A_{i j} \partial_{k m}^{2} f_{j}(b)\right|\right) \rho,
\end{aligned}
$$

since $c-\bar{x} \in \overline{B_{\rho}(0)}$. The result follows by taking the supremum on all $b \in \overline{B_{\rho_{*}}(\bar{x})}$.

The 3-tensor $D_{r}^{2} f$ is given component-wise by

$$
\partial_{l j}^{2} f_{i}= \begin{cases}\frac{3 m_{i}}{\sqrt{2} r_{i}^{4}} \zeta_{\ell}+\frac{6 m_{0}}{r_{i}^{4}}+\sum_{\substack{j=1 \\ j \neq i}}^{n} \frac{3 m_{j}}{2} \sum_{k=0}^{\ell-1} \frac{\left(r_{i}-r_{j} \cos \theta_{k}\right)\left(4 r_{i}^{2}-r_{j}^{2}-8 r_{i} r_{j} \cos \theta_{k}+5 r_{j}^{2} \cos 2 \theta_{k}\right)}{\left.\left(r_{i}^{2}+r_{j}^{2}-2 r_{i} r_{j} \cos \theta_{k}\right)\right)^{7 / 2},}, l=j=i, \\ -\frac{3 m_{j}}{4} \sum_{k=0}^{\ell-1} \frac{\left(r_{i}\left(8 r_{i}^{2}+23 r_{j}^{2}\right) \cos \theta_{k}-r_{j}\left(20 r_{i}^{2}+2 r_{j}^{2}+\left(4 r_{i}^{2}+6 r_{j}^{2}\right) \cos 2 \theta_{k}-r_{i} r_{j} \cos 3 \theta_{k}\right)\right)}{\left.\left(r_{i}^{2}+r_{j}^{2}-2 r_{i} r_{j} \cos \theta_{k}\right)\right)^{7 / 2},}, l \neq j=i \text { or } j \neq l=i, \\ -\frac{3 m_{j}}{4} \sum_{k=0}^{\ell-1} \frac{\left(r_{j}\left(8 r_{j}^{2}+23 r_{i}^{2}\right) \cos \theta_{k}-r_{i}\left(20 r_{j}^{2}+2 r_{i}^{2}+\left(4 r_{j}^{2}+6 r_{i}^{2}\right) \cos 2 \theta_{k}-r_{i} r_{j} \cos 3 \theta_{k}\right)\right)}{\left.\left(r_{i}^{2}+r_{j}^{2}-2 r_{i} r_{j} \cos \theta_{k}\right)\right)^{7 / 2},}, l=j \neq i, \\ 0, & l \neq j \neq i .\end{cases}
$$


We unfold $D_{r}^{2} f$ and represent it by the $n \times n^{2}$ matrix $B(b)=\left(B_{1}(b)|\cdots| B_{n}(b)\right)$, where

$$
B_{i}=\left(\begin{array}{ccccc}
-\partial_{1 i}^{2} \frac{F_{1}(b)}{m_{1}} & \ldots & -\partial_{j i}^{2} \frac{F_{1}(b)}{m_{1}} & \ldots & -\partial_{n i}^{2} \frac{F_{1}(b)}{m_{1}} \\
\vdots & & \vdots & & \vdots \\
-\partial_{1 i}^{2} \frac{F_{k}(b)}{m_{k}} & \ldots & -\partial_{j i}^{2} \frac{F_{k}(b)}{m_{k}} & \ldots & -\partial_{n i}^{2} \frac{F_{k}(b)}{m_{k}} \\
\vdots & & \vdots & & \vdots \\
-\partial_{1 i}^{2} \frac{F_{n}(b)}{m_{n}} & \ldots & -\partial_{j i}^{2} \frac{F_{n}(b)}{m_{n}} & \ldots & -\partial_{n i}^{2} \frac{F_{n}(b)}{m_{n}}
\end{array}\right), \quad i=1, \ldots, n
$$

such that the $Z_{2}$ bound from Lemma 6.5 is obtained by

$$
Z_{2}=\sup _{b \in \overline{B_{\rho_{*}}(\bar{r})}}\|A B(b)\|_{\infty}
$$

\subsection{Numerical Experimentations with Circles of Equal Mass}

The rigorous numerical proofs have been done with $\lambda=-1$ for all $n \leq 100, \ell \leq 200$, $m_{0}=0$ and $m_{1}=\ldots=m_{n}$. Additionally, our numerical investigations lead us to believe that the $n \times \ell$ and $n \times \ell+1$ spiderweb central configurations not only exist, but are unique in the sense of Sect. 2.1. Saari stated the same claim in his papers $[10,11]$.

\section{Mass Distribution}

The numerical approach allows quantitative insights on spiderweb central configurations. All the profiles studied in this section are validated by applying Theorem 6.1 to each spiderweb central configuration.

For this purpose we introduce some invariants of the configurations. The first is the relative spacing between consecutive circles (see Fig. 3)

$$
a_{i}=a_{i}(\ell)=\frac{r_{i+1}(\ell)-r_{i}(\ell)}{r_{1}(\ell)}
$$

The second is the relative width of a spiderweb central configuration given by (see Fig. 4)

$$
b=b(\ell)=\sum_{i=1}^{n-1} a_{i}(\ell)=\frac{r_{n}(\ell)-r_{1}(\ell)}{r_{1}(\ell)}
$$

Depending on the context, we write explicitly the dependence on $\ell$.

Conjecture 7.1 (See Fig. 3) For circles of equal mass and any $n \in \mathbb{N}, \ell \in \mathbb{N}_{\geq 2}$, the sequence $\left\{a_{i}\right\}_{1 \leq i \leq n-1}$ is convex. When $\ell=2$ and only in this case, the sequence is strictly increasing. 


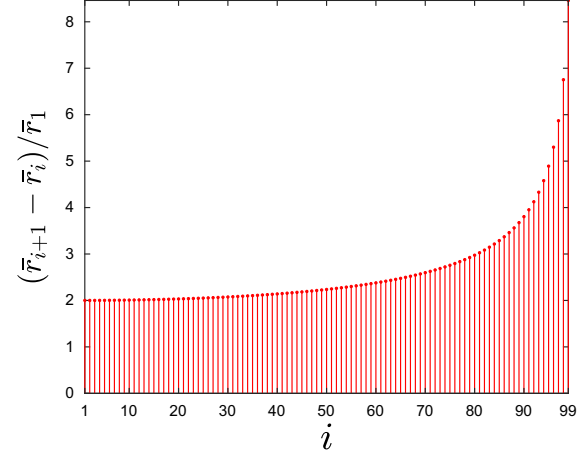

(a) $\ell=2$

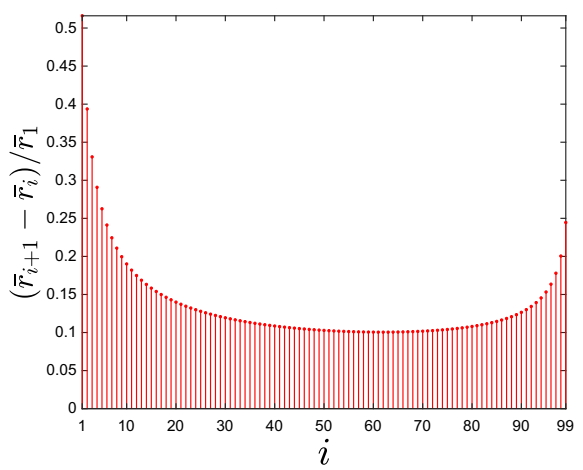

(c) $\ell=100$

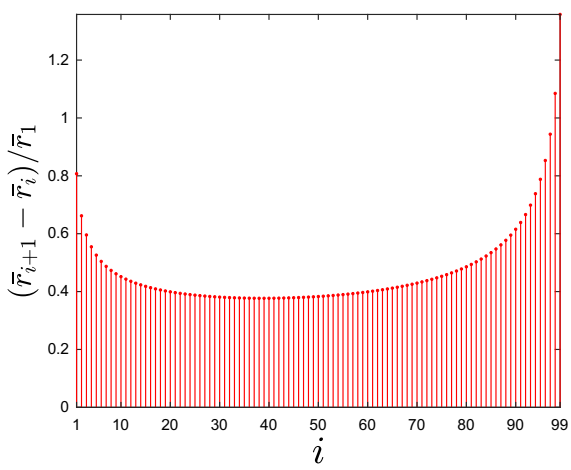

(b) $\ell=6$

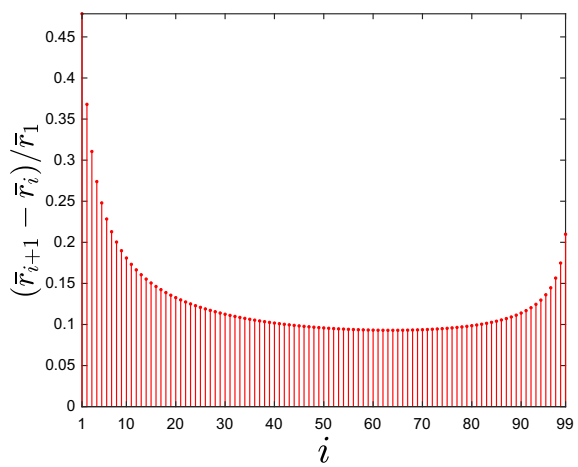

(d) $\ell=200$

Fig. 3 Spacing between consecutive circles of spiderweb central configurations with circles of equal mass and $\lambda=-1, n=100, m_{0}=0$ for different values of $\ell$

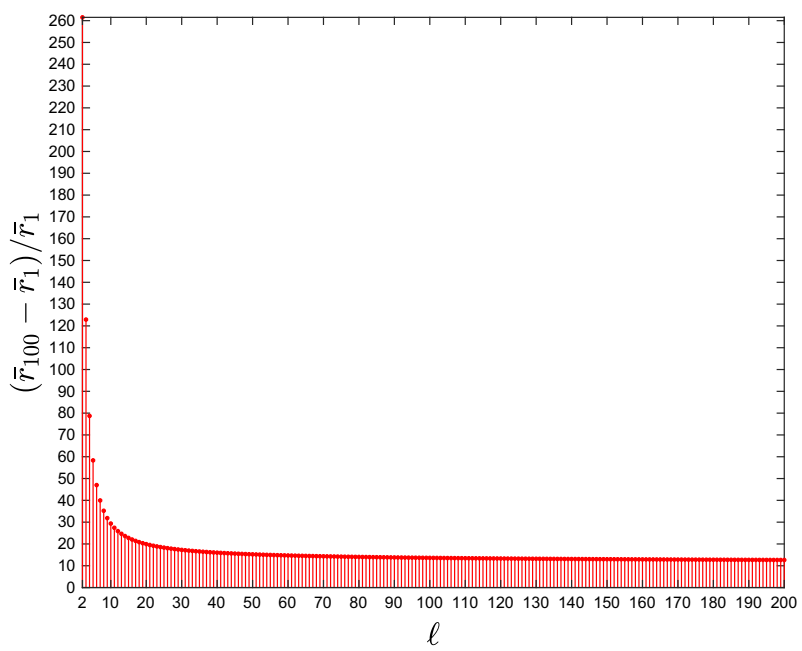

Fig. 4 Evolution of the relative width with respect to $\ell$ of spiderweb central configurations with circles of equal mass and $\lambda=-1, n=100, m_{0}=0$ 
Moreover, let $a_{i *}$ be the maximum of the $a_{i}$. From the convexity we know that $i^{*} \in\{1, n-1\}$ and, a priori, we cannot exclude that $a_{1}=a_{n-1}$. Numerically however we have never seen $a_{1}=a_{n-1}$. Hence we could have a unique $i^{*}$. There exists an increasing function $\mu: \mathbb{N} \longrightarrow \mathbb{N}$ such that

$$
i^{*}= \begin{cases}1, & \ell \geq \mu(n), \\ n-1, & \ell<\mu(n) .\end{cases}
$$

Numerically $\mu(n)$ seems small compared to $n$. Thus, $i^{*}=1$ whenever $\ell \geq n$.

Figure 4 shows that the relative width $b$ decreases with $\ell$ : the spiderweb central configurations form denser cluster.

Let $\chi(\eta)=\#\left\{j \in\{1, \ldots, n\}: r_{j} \leq \eta\right\}$. The mass distribution of a spiderweb central configuration, with $n$ circles, $m_{0}=0$ and $\ell$ equal masses per circle $m_{1}, \ldots, m_{n}$, is given by

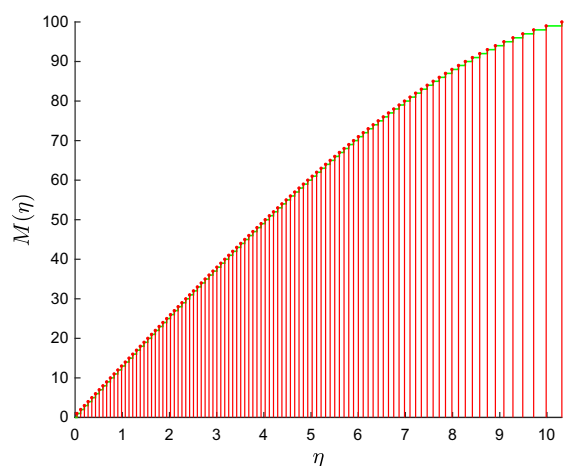

(a) $\ell=2$

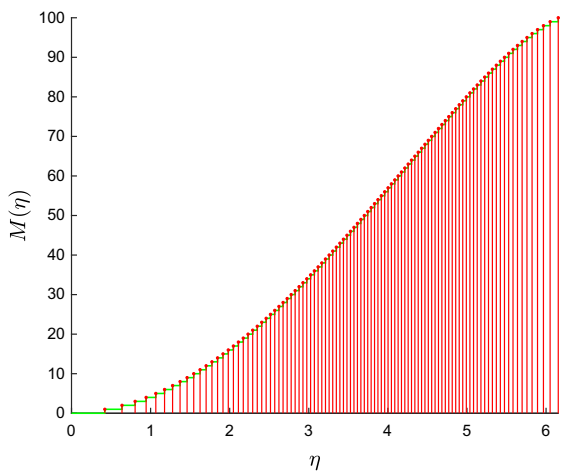

(c) $\ell=100$

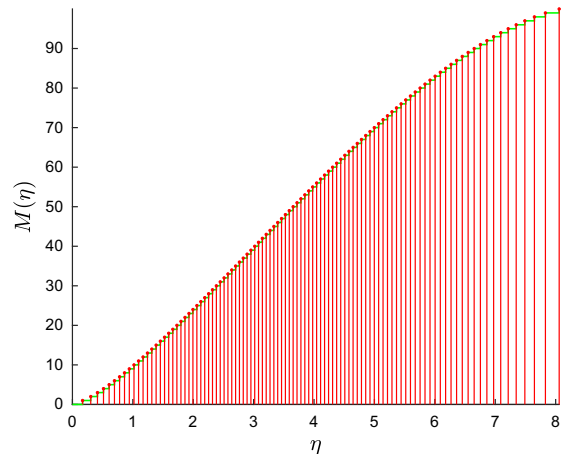

(b) $\ell=6$



(d) $\ell=200$

Fig. 5 Mass distribution $M(\eta)$ of spiderweb central configurations with $\lambda=-1, n=100,\left(m_{0}, m\right)=$ $(0,1 / \ell, \ldots, 1 / \ell)$ and different values of $\ell$ 


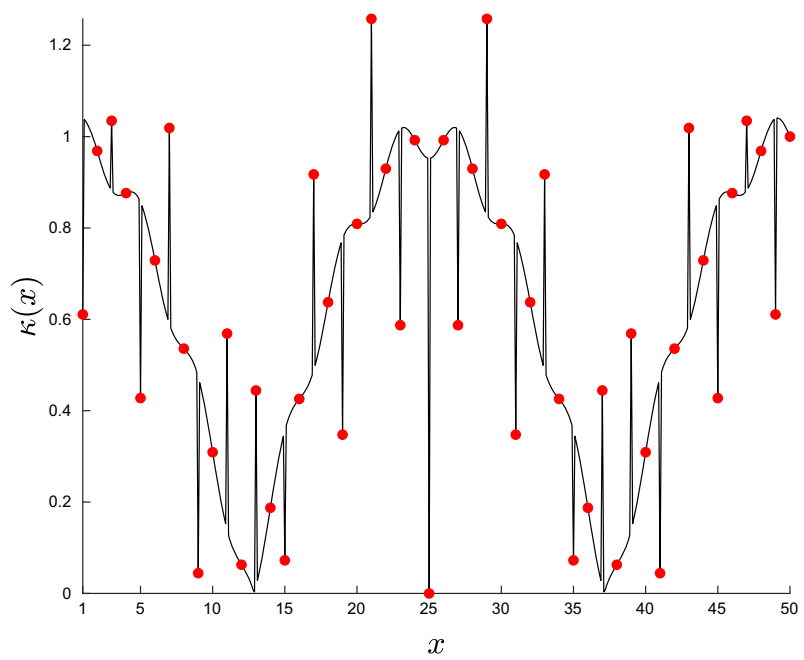

Fig. 6 Graph of $\kappa(x)=\left|\frac{\sin (21 \pi(x-25))}{42 \sin \left(\frac{\pi(x-25)}{2}\right)}+\cos \left(\frac{\pi x}{25}\right)\right|$ where $m_{i}=\kappa(i)$ for $i=1, \ldots, 50(\bullet)$

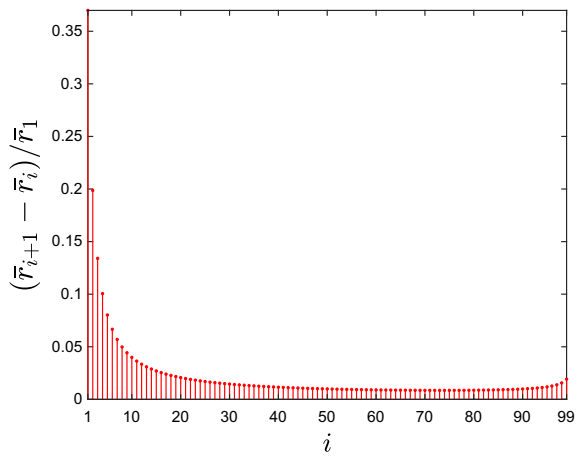

(a) Sequence $\left\{a_{i}\right\}$

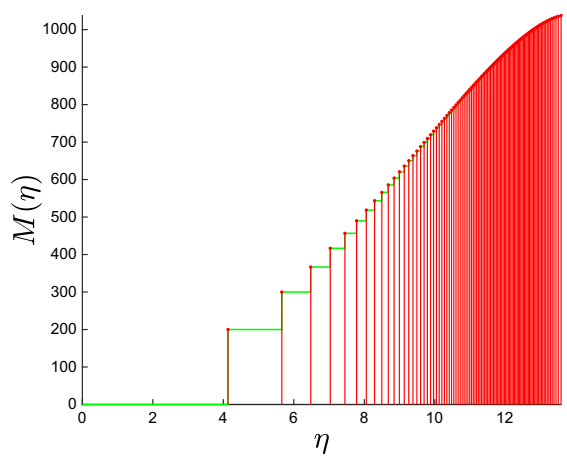

(c) Corresponding $M(\eta)$

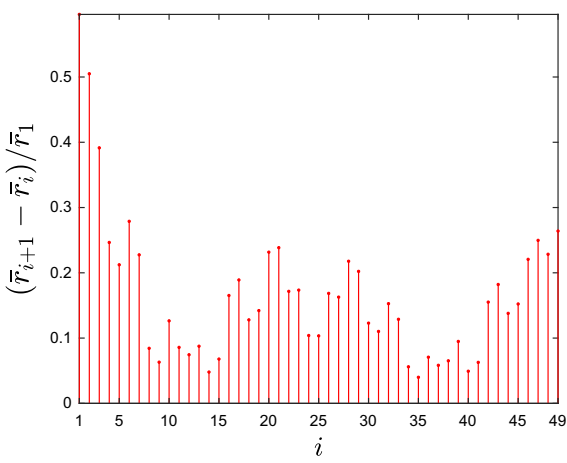

(b) Sequence $\left\{a_{i}\right\}$

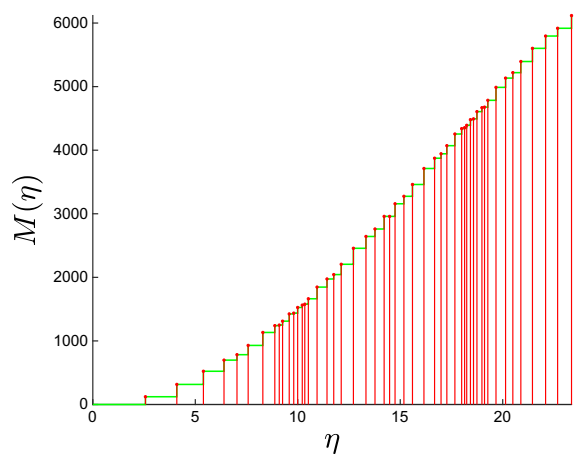

(d) Corresponding $M(\eta)$

Fig. 7 For $\lambda=-1$, a, b represent the spacing between consecutive circles for the spiderweb central configurations with $n=100, \ell=200,\left(m_{0}, m\right)=(0,1,1 / 2, \ldots, 1 / 100)$ and $n=50, \ell=200,\left(m_{0}, m\right)=$ $(0, \kappa(1), \ldots, \kappa(n))$ respectively, while $\mathbf{c}, \mathbf{d}$ show their respective mass distribution 


$$
M(\eta)=\ell \sum_{i=1}^{\chi(\eta)} m_{i}
$$

By definition, $M(\eta)$ is given for the values of $\lambda$ and $m_{1}, \ldots, m_{n}$ chosen beforehand. The mass distribution explored in Fig. 3 is shown on Fig. 5 in the case of equal masses. What is remarkable is that while the sequence $\left\{a_{1}, \ldots, a_{n}\right\}$ can have a very wild behavior when the sequence $\left\{m_{1}, \ldots, m_{n}\right\}$ is irregular, the mass distribution $M(\eta)$ looks very regular (see Figs. 6, 7).

This suggests that the general shape of the mass distribution is intrinsic to the spiderweb central configurations. Heuristically, this property yields a new perspective on the uniqueness of these central configurations. Figures 6 and 7 illustrate this: the invariant relative spacing $a_{i}$ defined in (7.1) becomes irrelevant, while the system compensates for lighter or heavier masses to reach the appropriate smooth mass distribution.

Hence, one might think of $M(\eta)$ as an alternative characteristic of a spiderweb central configuration, whose properties deserve further study.

Acknowledgements We are grateful to Jean-Philippe Lessard for helpful discussions and inputs on the computational part of the paper.

Open Access This article is distributed under the terms of the Creative Commons Attribution 4.0 International License (http://creativecommons.org/licenses/by/4.0/), which permits unrestricted use, distribution, and reproduction in any medium, provided you give appropriate credit to the original author(s) and the source, provide a link to the Creative Commons license, and indicate if changes were made.

\section{References}

1. Benet, L., Sanders, D.P.: IntervalArithmetic, Julia package version 0.14 (2013), https://juliaintervals. github.io/IntervalArithmetic.j1/latest/

2. Corbera, M., Delgado, J., Llibre, J.: On the existence of central configurations of $p$ nested $n$-gons. Qual. Theory Dyn. Syst. 8, 255-265 (2009)

3. Hénot, O.: Proofs and animations for spiderweb configurations, http://dms.umontreal.ca/ rousseac/ spiderweb.zip

4. Hungria, A., Lessard, J.P., Mireles, J.J.D.: Rigorous numerics for analytic solutions of differential equations: the radii polynomial approach. Math. Comput. 85, 1427-1459 (2016)

5. Llibre, J., Mello, L.F.: Triple and quadruple nested central configurations for the planar $n$-body problem. Physica D 238, 563-571 (2009)

6. Maxwell, J.C.: On the Stability of the Motion of Saturn's Ring. Macmillan and Co, London (1859)

7. Moeckel, R., Simo, C.: Bifurcations of spatial central configurations from planar ones. SIAM J. Math. Anal. 26, 978-998 (1995)

8. Montaldi, J.: Existence of symmetric central configurations. Celest. Mech. Dyn. Astron. 122, 405-418 (2015)

9. Moulton, F.R.: The straight line solutions of the problem of $N$ bodies. Ann. Math. Second Ser. 12, $1-17$ (1910)

10. Saari, D.: Mathematics and the "Dark Matter" puzzle. Am. Math. Mon. 122, 407-423 (2015)

11. Saari, D.: $N$-body solutions and computing galactic masses. Astron. J. 149, 1-6 (2015)

12. Zhang, S., Xie, Z.: Nested regular polygon solutions of the $2 N$-body problem. Phys. Lett. A 281, 149-154 (2001)

13. Zhang, S., Zhou, Q.: Periodic solutions for the $2 N$-body problems. Proc. Am. Math. Soc. 131, 2161$2170(2003)$

Publisher's Note Springer Nature remains neutral with regard to jurisdictional claims in published maps and institutional affiliations. 